\title{
Classification and Solving of Merge Patterns in Geometric Constraint Solving
}

\author{
Xiao-Shan Gao $^{1}$ and Gui-Fang Zhang ${ }^{1,2}$ \\ Institute of System Science, AMSS, Chinese Academy of Sciences ${ }^{1}$ \\ Beijing 100080, China \\ Department of Mathematics, Capital Normal University ${ }^{2}$ \\ Beijing 100037, China \\ (xgao,gfzhang)@mmrc.iss.ac.cn
}

\begin{abstract}
A basic idea of geometric constraint solving is to divide a large geometric constraint problem into several subproblems according to certain patterns and then to merge these subproblems to obtain a solution to the original problem. In this paper, we give a classification of the basic merge patterns in $2 \mathrm{D}$ when solving a constraint problem with generalized construction sequences. We obtain the analytical solutions to all eleven cases of merge patterns which is to assemble two rigids connected with three constraints. We also give conditions to solve these patterns with ruler and compass constructions.
\end{abstract}

Keywords. Geometric constraint solving, parametric CAD, generalized construction sequence, basic merge pattern, assembly.

\section{Introduction}

Geometric constraint solving is one of the key techniques in parametric CAD, which allows the user to make modifications to existing designs by changing parameter values. There are four major approaches to geometric constraint solving: the numerical approach[14, 18], the symbolic approach $[6,11]$, the rule-based approach[1, 5, 12, 21] and the graph-based approach $[2,9,10,15,16,17]$.

One of the effective paradigms of geometric constraint solving is to decompose a wellconstrained problem into a generalized construction sequence (GCS)

$$
C_{1}, C_{2}, \cdots, C_{n}
$$

where each $C_{i}$ is a set of geometric elements, such that

1. The geometric primitives in $C_{i}$ only have constraints with primitives in $C_{1}, \cdots, C_{i-1}$, which is called the external constraint of the geometric primitives in $C_{i}$;

2. $\bigcup_{k=1}^{i} C_{k}$ is a rigid for each $1 \leq i \leq m$;

3. No proper subsets of $C_{i}$ satisfy conditions 1 and 2 .

Latham and Middleditch are the first to propose an algorithm to find a generalized construction sequence [15]. Their method is based on the maximal b-matching from graph 
theory. Dong et al proposed similar algorithms based on degree of freedom analysis and translate an undirected graph to directed graph from graph theory [3]. Chen et al proposed an improved version of Dong's algorithm [2]. Hoffmann et al gave an algorithm to find rigids in a constraint problem [9]. From this, it is easy to obtain a GCS.

After a GCS is obtained, we need to compute the position of $C_{i}$ relative to $B_{i}=\bigcup_{k=1}^{i-1} C_{k}$. This is usually done with numerical methods such as the Newton-Raphson method [18], the homotopic method[14], and the optimize method[8]. This kind of methods although fast is generally not robust and can be used to find one solution only. Since geometric constraint solving is often used in the conceptual design, it would be desirable to find all the solutions of the problem. A locus intersection methods to find all solutions is proposed in [7]. This method is a hybrid method of geometric construction and numerical search. On the other hand, we may give a fast and stable way to find all the solutions if we may solve the problem with geometric or symbolic methods. In this paper, we propose such a method to compute the position of $C_{i}$ relative to $B_{i}$ when both $C_{i}$ and $B_{i}$ are rigids.

In Section 2, we give a classification of the merge patterns between $B_{i}$ and $C_{i}$ according to the number of constraints between $B_{i}$ and $C_{i}$. This $2 \mathrm{D}$ classification reveals the basic constraint problems need to be solved in order to solve the GCS. These patterns may also be considered as basic configurations as proposed in [7] in that they are the smallest problems to be solved in solving a GCS. We also show that one of the interesting pattern can be found in quadratic complexity.

When both $B_{i}$ and $C_{i}$ are rigids, the problem may be considered as an assembly problem. We need to assemble two rigids according to three constraints. This is the most general 2D assembly problem for two rigids, because we need three independent conditions to determine the position of a rigid. We divide this problem into two cases:

1. dda: there exist one angle constraint and two distance constraints between $B_{i}$ and $C_{i}$.

2. ddd: there exist three distance constraints between $B_{i}$ and $C_{i}$.

In [16], this type of problems is used as basic problems to solve general constraint problems. In [7], these basic merge patterns are solved with the locus intersection method. In Section 3 , we give closed form solutions to all the eleven dda and ddd cases. In particular, we show that the dda case can be solved with ruler and compass construction, which is fast and stable.

It is always desirable to solve a constraint problem by ruler and compass construction. In [16], it is said that the problem of assembling two rigids can not be solved with ruler and compass when the sum of external constraints for the vertex in $C_{i}$ is less than its degree of freedom. In this paper, we give a detailed analysis of this problem. We proved that the dda problem is always ruler and compass constructible. The ddd problem can be divided into ten cases. Among these cases two are ruler and compass constructible and the rest can not be solved with ruler and compass. In Section 4, conditions for ruler and compass constructions in the general case are also proposed.

Most of the results in this paper have been extended to 3D case, which will be presented in another paper.

\section{Basic Merge Patterns}


We consider two types of geometric primitives: points and lines in the Euclidean plane and two basic types of constraints: the angle constraints and the distance constraints. We use a constraint graph to represent a constraint problem. The vertices of the graph represent the geometric primitives and the edges represent the constraints. Figure 2 is an example of graph representation for a constraint problem in Figure 1.

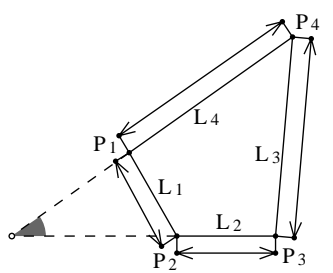

Figure 1. Lengths of four edges and angle $\left(\mathbf{L}_{2}, \mathbf{L}_{4}\right)$ are given

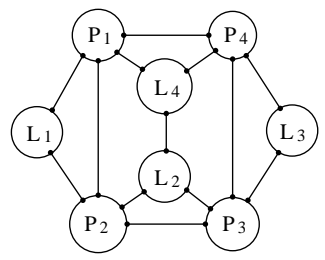

Figure 2. The graph representation

For an edge or constraint $e$ in the graph, let $\operatorname{DOC}(e)$ be the valence of $e$, which is the number of scalar equations required to define that constraint. Without loss of generality, we assume that every constraint has valence one.

For a geometric primitive $o$ in a constraint problem, let $\mathrm{DOF}(o)$ be the degree of freedoms for $o$ and $\operatorname{DEG}(o)$ the number of constraints involving $o$. For a set of geometric primitives $O$ in a constraint problem, let $\operatorname{DOF}(O)=\sum_{o \in O} \operatorname{DOF}(o)$ and $\operatorname{DEG}(O)$ the number of constraints among primitives in $O$.

As mentioned in Section 1, a constraint problem can be decomposed into a generalized construction sequence

$$
C_{1}, C_{2}, \cdots, C_{n}
$$

We call the type of dependency of $C_{i}$ on $C_{1}, \cdots, C_{i-1}$ basic merge patterns. Let

$$
\mathcal{B}=\bigcup_{k=1}^{i-1} C_{k}, \mathcal{U}=C_{i}
$$

The problem is to determine $\mathcal{U}$ assuming that $\mathcal{B}$ is known. The problem is shown in Figure $3-(\mathrm{a})$.

The sum of $\operatorname{DOC}(e)$ for all edges $e$ between $\mathcal{B}$ and $\mathcal{U}$ describes an important natural of the merging step, and is called the connection number of the problem, denoted by $\operatorname{CN}(\mathcal{B}, \mathcal{U})$.

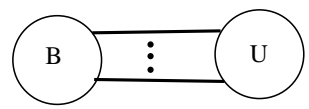

(a)

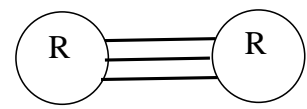

(b)

Figure 3

In what below, we will analyze the structures of the basic merging patterns and show how to position $\mathcal{U}$ relative to $\mathcal{B}$ when the geometric entities are only points and lines. We call $\mathcal{B}$ and $\mathcal{U}$ the base object and the dependent object, respectively. In what below, an edge only represents a constraint of valency one. Let graph $\mathcal{U}=(V, E)$, where $V$ is the set of vertices and $E$ is the set of edges. 
Theorem 2.1 We have: $2 \leq C N(\mathcal{B}, \mathcal{U}) \leq|V|$.

Proof: Since $V$ contains at least one geometric primitive and $\mathcal{B}$ is a rigid, $|C N(\mathcal{B}, \mathcal{U})| \geq 2$. The definition of GCS guarantees that $\mathcal{U}$ satisfies the following three conditions [15].

1. A vertex $v$ in $V$ represents a point or a line. Hence $\operatorname{DOF}(v)=2$;

2. If $|V|>1$ then for every vertex $v$ in $V$ there exists at least one constraint between $v$ and a primitive in $V$. Otherwise, $v$ can be determined by $\mathcal{B}$ alone, which contradicts to the minimum property of $V$ (the third condition on the definition of GCS).

3. $\mathcal{U}$ is strongly connected.

Due to the second condition above, there exist at least $2 \times \frac{|V|}{2}=|V|$ constraints between primitives in $E$, i.e.

$$
\operatorname{DEG}(V) \geq|V| \text {. }
$$

Since both $\mathcal{B}$ and $\mathcal{U}$ are rigids, we need exactly

$$
\operatorname{DOF}(V)=\Sigma_{v \in V} \operatorname{DOF}(v)=2|V|
$$

constraints to determine $\mathcal{U}$. In other words, we have

$$
\mathrm{CN}(\mathcal{B}, \mathcal{U})+\operatorname{DEG}(V)=2|V|
$$

Thus $\operatorname{CN}(\mathcal{B}, \mathcal{U})=2|V|-\operatorname{DEG}(V) \leq|V|$.

\section{I}

The computation of $\mathcal{U}$ with respect to $\mathcal{B}$ can be divided into the following three cases.

1. If $\mathrm{CN}(\mathcal{B}, \mathcal{U})=2, \mathcal{U}$ should consist of one geometric element $o$, and $o$ can be constructed explicitly with ruler and compass.

2. If $\mathrm{CN}(\mathcal{B}, \mathcal{U})=3, \mathcal{U}$ should have $2|V|-3$ constraints between primitives in $V$. Hence $\mathcal{U}$ is a rigid itself. It may be considered as to assembly two rigids according to three constraints, as shown by Figure 3-(b). We give complete analytical solution to this case in Section 3.

3. If $\mathrm{CN}(\mathcal{B}, \mathcal{U})>3$, the problem becomes more complicated. Now $\mathcal{U}$ is not a rigid anymore. We need to use the constraints inside $\mathcal{U}$ and those between $\mathcal{U}$ and $\mathcal{B}$ to determine $\mathcal{U}$. As an example, the basic merging patterns for the case $|\mathcal{U}|=5$ is shown in Figure 4.

In what below, we use circles to represent the vertices with two degrees of freedom, circles labelled $\mathbf{R}$ to represent the rigids, and the thin lines to represent the constraints between $\mathcal{B}$ and $\mathcal{U}$.

When $\operatorname{CN}(\mathcal{B}, \mathcal{U})$ is maximal, the merge patterns of $\operatorname{CN}(\mathcal{B}, \mathcal{U})=3, \operatorname{CN}(\mathcal{B}, \mathcal{U})=4$ and $\mathrm{CN}(\mathcal{B}, \mathcal{U})=5$ are shown in Figure 5 .

In Figure 5, for any vertex $v$ in $\mathcal{U}, \operatorname{DEG}(v)$ is equal to 3 , and $v$ is adjacent to three different vertexes, where one is in $\mathcal{B}$ and the rest in $\mathcal{U}$. Because of this property, we may design the following algorithm to identify this case easily. 

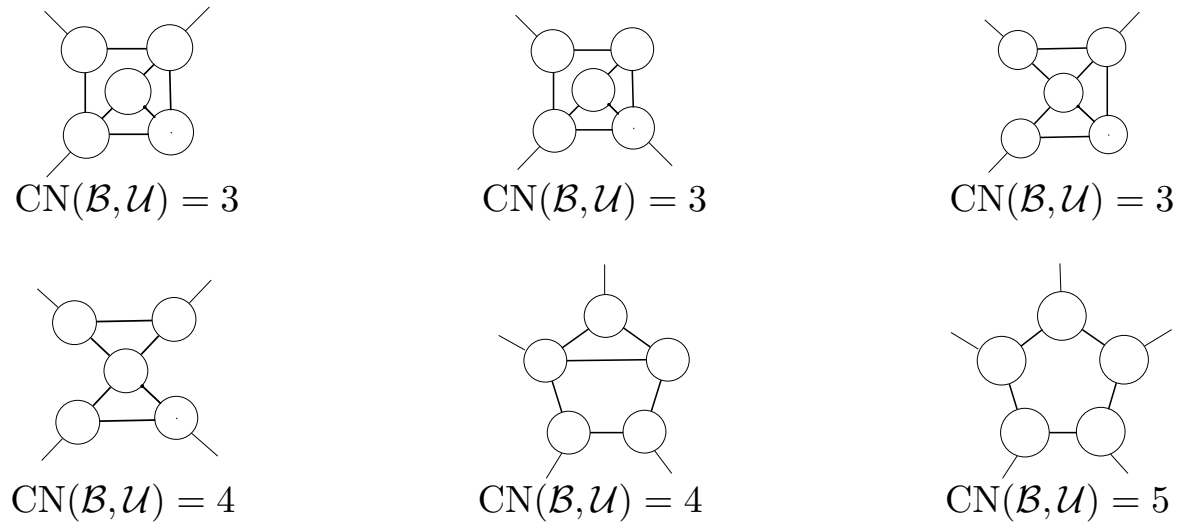

Figure 4. Basic merge patterns of five primitives
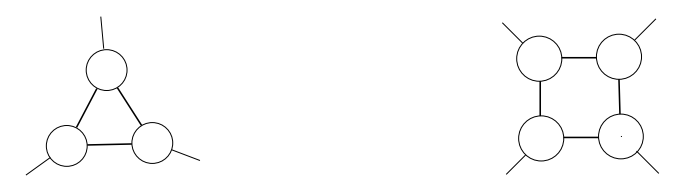

$\mathrm{CN}(\mathcal{B}, \mathcal{U})=3$

$$
\mathrm{CN}(\mathcal{B}, \mathcal{U})=4
$$

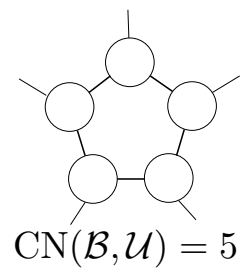

Figure 5. Merge patterns when $\mathrm{CN}(\mathcal{B}, \mathcal{U})$ is maximal

1. We first delete those vertices satisfying $\operatorname{DEG}(v) \leq 2$ until no vertices satisfying this condition. These vertices can be constructed explicitly.

2. We search the graph to find vertices $v_{1}, \cdots, v_{m}$ satisfying $\operatorname{DEG}\left(v_{i}\right)=3, i=1, \cdots, m$ and there is one edge between $v_{i}$ and $v_{i+1}$. These vertices may serve as a set in the GCS. Delete them and goto step one.

3. If no such vertices exist, the algorithm terminates without finding a GCS.

This algorithm is of quadratic complexity in terms of the number of vertices and the number of edges of the constraint graph. In each time to execute step 1 or step 2 , we will delete at least one vertices. Therefore, we will execute step 1 or step 2 for at most $n$ times, where $n$ is the number of the vertices. In step 2, we need to search a set of vertices which need $O(e+n)$ steps, where $e$ is the number of edges. Totally, we need at most $O\left(n^{2}+e * n+e^{2}\right)$ steps.

In Figure $4, \mathcal{U}$ is treated as a set of geometric elements. We may further to decompose $\mathcal{U}$ as a set of rigids if possible. This will simplify the problem greatly. In Figure 6, the six merge patterns for case $|\mathcal{U}|=5$ in Figure 4 are simplified to three patterns. Actually, these patterns represent more cases, because the rigids in these patterns may be of any size. The first two cases are used in [16] as basic patterns to solve constraint problems.

\section{Analytic Solution to Basic Merge Patterns: Case $\operatorname{CN}(\mathcal{B}, \mathcal{U})=3$}

When $\mathrm{CN}(\mathcal{B}, \mathcal{U})=3$, the basic pattern is shown in Figure 3(b). Now, both $\mathcal{B}$ and $\mathcal{U}$ are rigids. Hence, it is may be considered as an assembly problem. The problem can be divided 

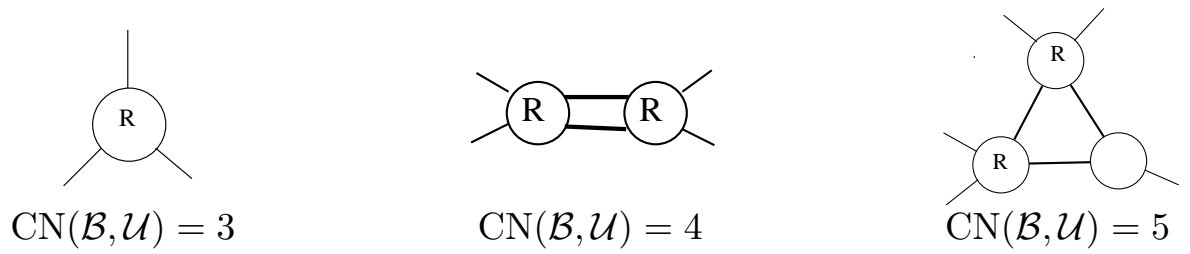

Figure 6. Merge patterns for rigids

into two cases: the ddd case in which all the three constraints between $\mathcal{B}$ and $\mathcal{U}$ are distance constraints and the dda case in which two of the constraints are distance and one constraint is an angle constraint. We cannot have more than one angle constraint due to the fact that a $2 \mathrm{D}$ rigid only need one angle constraint to determine its direction. In what below, we will give analytic solutions to the dda and ddd cases. Let us state the problem precisely as follows: $\mathcal{B}$ is a rigid whose position is fixed. $\mathcal{U}$ is a rigid with three constraints with $\mathcal{B}$. We need to position the rigid $\mathcal{U}$ relative to the rigid $\mathcal{B}$.

\subsection{The Case of dda}

We make use of the fact that rotation constraints can violate previous imposed distance constraints while translation doesn't violate previous imposed angular constraints. We impose the angular constraint first to remove the rotation degree of freedom. This will give us a pure geometric solution based on ruler and compass construction.

\section{Imposing Angular Constraint}

Let line $\mathbf{L}_{\mathbf{1}}$ be in $\mathcal{B}$, the base object set, and line $\mathbf{L}_{\mathbf{2}}$ in $\mathcal{U}$, the dependent object set. Assume that the parametric equations of $\mathbf{L}_{\mathbf{1}}$ and $\mathbf{L}_{\mathbf{2}}$ before the constraint is satisfied are

$$
\begin{aligned}
& \mathbf{L}_{\mathbf{1}}: \mathbf{P}=\mathbf{P}_{\mathbf{1}}+u_{1} \mathbf{s}_{\mathbf{1}} \\
& \mathbf{L}_{\mathbf{2}}: \mathbf{P}=\mathbf{P}_{\mathbf{2}}+u_{2} \mathbf{s}_{\mathbf{2}}
\end{aligned}
$$

where $\mathbf{P}=(x, y), \mathbf{s}_{\mathbf{1}}=\left(l_{1}, m_{1}\right),\left|\mathbf{s}_{\mathbf{1}}\right|=1, \mathbf{s}_{\mathbf{2}}=\left(l_{2}, m_{2}\right),\left|\mathbf{s}_{\mathbf{2}}\right|=1, \mathbf{s}_{\mathbf{1}} \times \mathbf{L}_{\mathbf{1}}=0, \mathbf{s}_{\mathbf{2}} \times \mathbf{L}_{\mathbf{2}}=0$, $\angle\left(\mathbf{L}_{\mathbf{1}}, \mathbf{L}_{\mathbf{2}}\right)=\angle\left(\mathbf{s}_{\mathbf{1}}, \mathbf{s}_{\mathbf{2}}\right)=\alpha$ and $\cos (\alpha)=l_{1} l_{2}+m_{1} m_{2}$.

If the rotation constraint is $\angle\left(\mathbf{L}_{1}, \mathbf{L}_{2}\right)=\beta, \mathbf{L}_{\mathbf{2}}$ should rotate angle $\theta=\beta-\alpha$ around $\mathbf{P}_{\mathbf{2}}$. After rotating angle $\theta$, the parametric equation of $\mathbf{L}_{\mathbf{2}}$ becomes $\mathbf{P}=\mathbf{P}_{\mathbf{2}}+u_{2} \mathbf{s}_{\mathbf{2}}^{\prime}$, where $\mathbf{s}_{\mathbf{2}}^{\prime}=\left(l_{2}, m_{2}\right) \times \mathbf{M}$, and $\mathbf{M}$ is

$$
\mathbf{M}=\left(\begin{array}{cc}
\cos \theta & \sin \theta \\
-\sin \theta & \cos \theta
\end{array}\right)
$$

After rotating an angle $\theta$, coordinates of geometric elements in the dependent object $(x, y)$ are transformed into $\left(x^{\prime}, y^{\prime}\right)=(x, y) \times \mathbf{M}$.

Imposing Distance Constraints

We will make use of the following definition and theorem in Kumar et al[13]. This theorem is also proposed by us in [5], under the name of 'translational' transformation.

Definition 3.1 The translation space of a point on the dependent object with respect to a distance constraint is the set of points to which the point can be moved to by translating the dependent object without violating the constraint. 
Theorem 3.2 If $\mathbf{R}^{X}(u)$ is the translation space of $\mathbf{P}_{0}$ on the dependent object with respect to a constraint $X$, the translation space of any other point $\mathbf{P}$ on the dependent object with respect to this constraint is $\mathbf{R}_{\mathbf{p}}{ }^{X}(u)=\mathbf{R}^{X}(u)+\left(\mathbf{P}-\mathbf{P}_{\mathbf{0}}\right)$, assuming that previously imposed angular constraints have eliminated all the rotation degree of freedom of the dependent object.

We may now give the following procedure for solving a dda problem.

Algorithm 3.3 The input is a dda constraint problem with $\mathcal{B}$ and $\mathcal{U}$ as the base and dependent objects. We will give a geometric construction procedure based on ruler and compass to solve the problem.

S1 We first fix the rotational degree of freedom of $\mathcal{U}$ by imposing the angular constraint.

S2 We will determine the translation spaces for the objects in the dependent objects according to the distance constraints left. This is divided into three cases.

1. If the distance constraint $\left|C_{0} P\right|=r_{0}$ is between a point $C_{0}$ in the base object and a point $P$ in the dependent object, then the translation space is the following circle

$$
R^{C}(\theta)=C_{0}+r_{0}(\cos \theta \mathbf{i}+\sin \theta \mathbf{j}) .
$$

2. The distance constraint $\left|L_{0} P\right|=r_{0}$ is between a line $L_{0}$ in the base object and a point $P$ in the dependent object. The translation space is a line if $r_{0}=0$. Otherwise it's two paralleling lines.

$$
R^{L}(u)=\mathbf{P}_{\mathbf{0}}+u \mathbf{I} \pm r_{0} \mathbf{m}
$$

where $P_{0}$ is a point on $L_{0}, \mathbf{I}$ is a unit vector parallel to $L_{0}$ and $\mathbf{m}$ is a unit vector perpendicular to $\mathbf{I}$.

3. The distance constraint $\left|P_{0} L\right|=r_{0}$ is between a point $P_{0}$ in the base object and a line $L$ in the dependent object. In this case, we take an arbitrary point $P$ in $L$ and will determine the translation space for $P$. The constraint $\left|P_{0} L\right|=r_{0}$ implies that $L$ is a tangent line of the circle with $P_{0}$ as center and as $r_{0}$ as the radius. Also, we know the direction of $L$ since the rotational degree of freedom of the dependent object is fixed. Then the position $L^{*}$ for $L$ can be easily determined by the two constraints. Since $P$ is a free point on line $L, L^{*}$ is the translation space for $L$.

S3 Since $\mathcal{U}$ has a fixed direction, we need only to compute the position $P^{*}$ of one point $P$ in $\mathcal{U}$. Then we need only to translate $\mathcal{U}$ by $P^{*}-P$ to move it to the correct position. By $\mathrm{S} 2$, for each distance constraint $d_{i}, i=1,2$, we have a point $P_{i}$ and a translation space $\mathbf{R}^{d_{i}}$ for $P_{i}$. By Theorem 3.2, the position for $P_{2}$ is the intersection of the following two translation spaces, which are either lines or circles.

$$
\left\{\begin{array}{l}
\mathbf{R}_{\mathbf{P}_{\mathbf{2}}}^{d_{1}}\left(u_{1}\right)=\mathbf{R}^{d_{1}}\left(u_{1}\right)+\left(\mathbf{P}_{\mathbf{2}}-\mathbf{P}_{\mathbf{1}}\right) \\
\mathbf{R}_{\mathbf{P}_{\mathbf{2}}}^{d_{2}}\left(u_{2}\right)=\mathbf{R}^{d_{2}}\left(u_{2}\right)
\end{array}\right.
$$


For the example in Figure 1, $\mathbf{L}_{\mathbf{1}}$ and $\mathbf{L}_{\mathbf{2}}$ can be constructed easily if the remaining objects are solved. After we delete $\mathbf{L}_{\mathbf{1}}$ and $\mathbf{L}_{\mathbf{3}}$ from the graph in Figure 2, the graph consists of two rigids: $\mathbf{P}_{\mathbf{1}} \mathbf{P}_{\mathbf{4}} \mathbf{L}_{\mathbf{4}}$ and $\mathbf{P}_{\mathbf{2}} \mathbf{P}_{\mathbf{3}} \mathbf{L}_{\mathbf{2}}$. Let $\mathbf{P}_{\mathbf{1}} \mathbf{P}_{\mathbf{4}} \mathbf{L}_{\mathbf{4}}$ be the dependent object and $\mathbf{P}_{\mathbf{2}} \mathbf{P}_{\mathbf{3}} \mathbf{L}_{\mathbf{2}}$ the base object respectively. We construct the translation space of distance constraint $d_{1}$ between point $\mathbf{P}_{\mathbf{1}}$ and $\mathbf{P}_{\mathbf{2}}$, which is circle $\mathbf{C}_{\mathbf{1}}$ shown in Figure 7 . Then we can construct the translation space for distance constraint $d_{2}$ between point $\mathbf{P}_{\mathbf{3}}$ and $\mathbf{P}_{\mathbf{4}}$, which is circle $\mathbf{C}_{\mathbf{3}}$ shown in Figure 7. According to Theorem 3.2 we can get the translation space of point $\mathbf{P}_{4}$ with respect constraint $d_{1}$, which is circle $\mathbf{C}_{\mathbf{2}}$. The intersection of circle $\mathbf{C}_{\mathbf{2}}$ and circle $\mathbf{C}_{\mathbf{3}}$ is the solution to point $\mathbf{P}_{\mathbf{4}}^{*}$. We can translate rigid $\mathbf{P}_{\mathbf{1}} \mathbf{P}_{\mathbf{4}} \mathbf{L}_{\mathbf{4}}$ along the vector $\mathbf{t}=\mathbf{P}_{\mathbf{4}}^{*}-\mathbf{P}_{\mathbf{4}}$ and position rigid $\mathbf{P}_{\mathbf{1}} \mathbf{P}_{\mathbf{4}} \mathbf{L}_{\mathbf{4}}$. The problem could have one or two solutions shown in Figure 7.

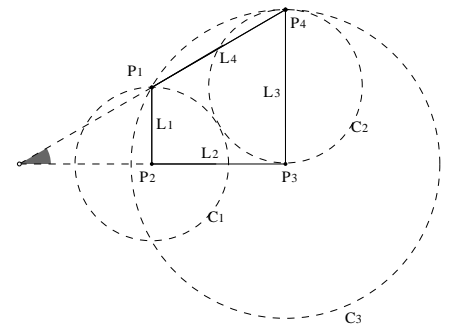

(a)

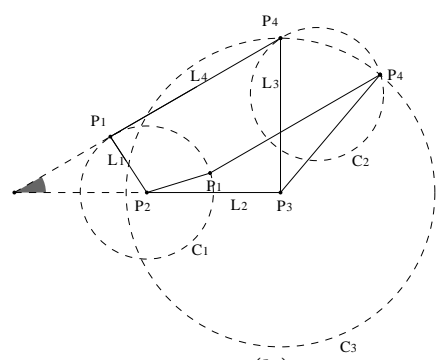

(b)

Figure 7. Geometric solution to the problem in Figure 1.

In Step S3, we need to find the intersection of lines/circles and lines/circle. The details of the computation is omitted. We would like to point out that when to compute the intersection of two lines, they will not parallel to each other. Otherwise, we will have conflict constraints. Let us consider the following case: the two distance constraints are $\left|P_{1} L_{1}\right|=d_{1}$ and $\left|P_{2} L_{2}\right|=d_{2}$ where $P_{i}$ are points in $\mathcal{U}$ and $L_{i}$ are lines in $\mathcal{B}$. Let us assume that $L_{1}$ is parallel to $L_{2}$. Since $P_{1}$ and $P_{2}$ are in a rigid $\mathcal{U}$ which has fixes direction, we may determine $\left|P_{1} L_{1}\right|$ from $\left|P_{2} L_{2}\right|$. Thus, the two constraints are conflict. Other cases can be treated similarly.

\subsection{The Case of ddd}

When the constraints between the base object and the dependent object are all distance constraints, there are altogether 10 different cases shown in Table 3.

\begin{tabular}{|l|l|l|}
\hline Constrain type & Vertex types in dependent object & Vertex types in base object \\
\hline PPP-LLL & three points & three lines \\
\hline PPP-LLP & three points & two lines, one point \\
\hline PPP-LPP & three points & one lines, two points \\
\hline LLL-PPP & three lines & three points \\
\hline LLP-PPP & two lines, one point & three points \\
\hline LPP-PPP & one line, two points & three points \\
\hline LPP-PLL & one line, two points & one point, two lines \\
\hline LLP-PPL & two lines, one point & two points, one line \\
\hline LPP-PLP & one line, two points & two points, one line \\
\hline PPP-PPP & three points & three points \\
\hline
\end{tabular}

Table 3 Ten Cases of the ddd Problem 
We can always get three points in the base object and three points in the dependent object, respectively. If the object involved is a line, we can take a point on it. Let three points in the base object be $\mathbf{B}_{\mathbf{1}}, \mathbf{B}_{\mathbf{2}}$ and $\mathbf{B}_{\mathbf{3}}$, and three points in the dependent object $\mathbf{D}_{\mathbf{1}}, \mathbf{D}_{\mathbf{2}}$ and $\mathbf{D}_{\mathbf{3}} . \mathbf{B}_{\mathbf{1}}, \mathbf{B}_{\mathbf{2}}$ and $\mathbf{B}_{\mathbf{3}}$ are not collinear, and $\mathbf{D}_{\mathbf{1}}, \mathbf{D}_{\mathbf{2}}$ and $\mathbf{D}_{\mathbf{3}}$ are not collinear, either.

Let $\mathbf{B}_{\mathbf{1}}$ be the origin of the fixed coordinate system in the base object, $\mathbf{B}_{\mathbf{1}} \mathbf{B}_{\mathbf{2}}$ the $\mathbf{x}$ axis. The coordinates of three points in the base object are $\mathbf{B}_{\mathbf{1}}=(0,0), \mathbf{B}_{\mathbf{2}}=\left(b_{1}, 0\right)$ and $\mathbf{B}_{\mathbf{3}}=\left(b_{2}, b_{3}\right)$.

Let the point $\mathbf{D}$ be the origin of the moving coordinate system in the dependent object. The coordinate of point $\mathbf{D}$ in the fixed coordinate system is $\mathbf{D}=\left(x_{3}, x_{4}\right)$, and point $\mathbf{D}$ is the foot of the perpendicular line from point $\mathbf{D}_{\mathbf{3}}$ to $\mathbf{D}_{\mathbf{1}} \mathbf{D}_{\mathbf{2}}$. Let $\angle\left(\mathbf{B}_{\mathbf{1}} \mathbf{B}_{\mathbf{2}}, \mathbf{D}_{\mathbf{1}} \mathbf{D}_{\mathbf{2}}\right)=\theta$, $x_{1}=\cos \theta, x_{2}=\sin \theta$. The moving coordinates of three points in the dependent object are $\mathbf{D}_{\mathbf{1}}=\left(-h_{1}, 0\right), \mathbf{D}_{\mathbf{2}}=\left(h_{2}, 0\right), \mathbf{D}_{\mathbf{3}}=\left(0, h_{3}\right)$, where $h_{1}, h_{2}, h_{3}$ are three nonnegative parameters. $\mathbf{D}_{1} \mathbf{D}_{2}$ is the x-axis of the moving coordinate system. Their coordinates in the fixed coordinate system are

$$
\left\{\begin{array}{l}
\mathbf{D}_{\mathbf{1 1}}=\left(-h_{1} x_{1}+x_{3},-h_{1} x_{2}+x_{4}\right) \\
\mathbf{D}_{\mathbf{2 2}}=\left(h_{2} x_{1}+x_{3}, h_{2} x_{2}+x_{4}\right) \\
\mathbf{D}_{\mathbf{3 3}}=\left(-h_{3} x_{2}+x_{3}, h_{3} x_{1}+x_{4}\right)
\end{array}\right.
$$

There exist at most three lines in the base object. Let the parametric equations of these lines be

$$
\left\{\begin{array}{l}
\mathbf{L}_{\mathbf{1}}: \mathbf{P}=\mathbf{B}_{\mathbf{3}}+u_{1} \mathbf{s}_{\mathbf{1}},\left(\mathbf{s}_{\mathbf{1}}=\left(l_{1}, m_{1}\right),\left|\mathbf{s}_{\mathbf{1}}\right|=1\right) \\
\mathbf{L}_{\mathbf{2}}: \mathbf{P}=\mathbf{B}_{\mathbf{2}}+u_{2} \mathbf{s}_{\mathbf{2}},\left(\mathbf{s}_{\mathbf{2}}=\left(l_{2}, m_{2}\right),\left|\mathbf{s}_{\mathbf{2}}\right|=1\right) \\
\mathbf{L}_{\mathbf{3}}: \mathbf{P}=\mathbf{B}_{\mathbf{1}}+u_{3} \mathbf{s}_{\mathbf{3}},\left(\mathbf{s}_{\mathbf{3}}=\left(l_{3}, m_{3}\right),\left|\mathbf{s}_{\mathbf{3}}\right|=1\right)
\end{array}\right.
$$

There exist at most three lines in base object. Let the parametric equations of these lines be

$$
\left\{\begin{array}{l}
\mathbf{L}_{\mathbf{0 1}}: \mathbf{P}=\mathbf{D}_{\mathbf{3}}+u_{1} \mathbf{s}_{\mathbf{1}},\left(\mathbf{s}_{\mathbf{1}}=\left(l_{1}, m_{1}\right),\left|\mathbf{s}_{\mathbf{1}}\right|=1\right) \\
\mathbf{L}_{\mathbf{0 2}}: \mathbf{P}=\mathbf{D}_{\mathbf{2}}+u_{2} \mathbf{s}_{\mathbf{2}},\left(\mathbf{s}_{\mathbf{2}}=\left(l_{2}, m_{2}\right),\left|\mathbf{s}_{\mathbf{2}}\right|=1\right) \\
\mathbf{L}_{\mathbf{0 3}}: \mathbf{P}=\mathbf{D}_{\mathbf{1}}+u_{3} \mathbf{s}_{\mathbf{3}},\left(\mathbf{s}_{\mathbf{3}}=\left(l_{3}, m_{3}\right),\left|\mathbf{s}_{\mathbf{3}}\right|=1\right)
\end{array}\right.
$$

Although we use the same $\mathbf{s}_{\mathbf{i}}$ in $\mathbf{L}_{\mathbf{i}}$ and $\mathbf{L}_{\mathbf{0}}(i=1,2,3)$, there will be no confusion, because the lines $\mathbf{L}_{\mathbf{i}}$ and $\mathbf{L}_{\mathbf{0} \mathbf{i}}(i=1,2,3)$ will not appear in the same case simultaneity. Let $\mathbf{L}_{\mathbf{0 1}}, \mathbf{L}_{\mathbf{0 2}}$, $\mathbf{L}_{\mathbf{0 3}}$ be three lines in the dependent object. Their parametric equations are

$$
\left\{\begin{array}{l}
\left.\mathbf{L}_{\mathbf{1 1}}: \mathbf{P}=\mathbf{D}_{\mathbf{3 3}}+u_{1} \mathbf{s}_{\mathbf{1 1}}, \mathbf{s}_{\mathbf{1 1}}=\left(l_{1} x_{1}-m_{2} x_{2}, l_{1} x_{2}+m_{1} x_{1}\right),\left|\mathbf{s}_{\mathbf{1 1}}\right|=1\right) \\
\left.\mathbf{L}_{\mathbf{2 2}}: \mathbf{P}=\mathbf{D}_{\mathbf{2 2}}+u_{2} \mathbf{s}_{\mathbf{2 2}}, \mathbf{s}_{\mathbf{2 2}}=\left(l_{2} x_{1}-m_{2} x_{2}, l_{2} x_{2}+m_{2} x_{1}\right),\left|\mathbf{s}_{\mathbf{2 2}}\right|=1\right) \\
\left.\mathbf{L}_{\mathbf{3 3}}: \mathbf{P}=\mathbf{D}_{\mathbf{1 1}}+u_{3} \mathbf{s}_{\mathbf{3 3}}, \mathbf{s}_{\mathbf{3 3}}=\left(l_{3} x_{1}-m_{3} x_{2}, l_{3} x_{2}+m_{3} x_{1}\right),\left|\mathbf{s}_{\mathbf{3 3}}\right|=1\right)
\end{array}\right.
$$

We use $|\mathbf{P L}|=d_{i}(i=1, \cdots 9 ; j=1,2,3)$ to denote the distance between point $\mathbf{P}$ and line $\mathbf{L}$, and $|\mathbf{P L}|=t_{i k}(i=1, \cdots 9 ; k=1,2)$ to denote the distance between two points. It is obvious that $d_{i j}$ and $t_{i k}$ should be nonnegative parameters.

Case PPP-LLL If the constraints are $\left|\mathbf{D}_{\mathbf{1 1}} \mathbf{L}_{\mathbf{3}}\right|=d_{13},\left|\mathbf{D}_{\mathbf{2 2}} \mathbf{L}_{\mathbf{2}}\right|=d_{12}$, and $\left|\mathbf{D}_{\mathbf{3 3}} \mathbf{L}_{\mathbf{1}}\right|=d_{11}$, we have the following equations:

$$
\left\{\begin{array}{l}
x_{1}^{2}+x_{2}^{2}-1=0 \\
\left|m_{3}\left(-h_{1} x_{1}+x_{3}\right)-l_{3}\left(-h_{1} x_{2}+x_{4}\right)\right|-d_{13}=0 \\
\left|m_{2}\left(h_{2} x_{1}+x_{3}-b_{1}\right)-l_{2}\left(h_{2} x_{2}+x_{4}\right)\right|-d_{12}=0 \\
\left|m_{1}\left(-h_{3} x_{2}+x_{3}-b_{2}\right)-l_{1}\left(h_{3} x_{1}+x_{4}-b_{3}\right)\right|-d_{11}=0
\end{array}\right.
$$


We may simplify the equation system as follows.

$$
\left\{\begin{array}{l}
x_{1}^{2}+x_{2}^{2}-1=0 \\
s_{4} x_{1}+m_{3} x_{3}+s_{5} x_{2}-l_{3} x_{4}+s_{1}=0 \\
s_{6} x_{1}+m_{2} x_{3}-s_{7} x_{2}-l_{2} x_{4}+s_{2}=0 \\
s_{8} x_{2}+m_{1} x_{3}-s_{9} x_{1}-l_{1} x_{4}+s_{3}=0
\end{array}\right.
$$

where $s_{1}= \pm d_{13}, s_{2}=-m_{2} b_{1} \pm d_{12}, s_{3}=-m_{1} b_{2}+l_{1} b_{3} \pm d_{11}, s_{4}=-m_{3} h_{1}, s_{5}=l_{3} h_{1}, s_{6}=$ $m_{2} h_{2}, s_{7}=l_{2} h_{2}, s_{8}=-m_{1} h_{3}, s_{9}=l_{1} h_{3}$. To find a closed form solution to the above equation system, we may use elimination methods such as the Gröbner basis method or the characteristic set method [22]. In this paper, the Wu-Ritt's characteristic set method is used. We may easily find the following triangular form for above equation system.

$$
\left\{\begin{array}{l}
C S[11]=z_{111} x_{4}+z_{112} x_{1}+z_{113} \\
C S[12]=z_{121} x_{3}+z_{122} x_{1}+z_{123} \\
C S[13]=z_{131} x_{2}+z_{132} x_{1}+z_{133} \\
C S[14]=z_{141} x_{1}^{2}+z_{142} x_{1}+z_{143}
\end{array}\right.
$$

where the $z_{i j k}$ are polynomials free of $x_{1}, \cdots, x_{4}$. The detailed expressions for coefficients $z_{i j}$ may be found in Appendix A, which may be found in http://www.mmrc.iss.ac.cn/ ${ }^{\sim}$ xgao/paper/2dst.ps.

The above analytical solutions reduce the problem to the solving of one quadratic equation and three linear equations. As a consequence, we know that this case is ruler and compass constructible. There are at most 16 solutions, since $s_{1}, s_{2}, s_{3}$ could take two opposite values and $C S[14]$ is of degree 2 .

Case PPP-LLP If the constraints are $\left|\mathbf{D}_{\mathbf{1 1}} \mathbf{B}_{\mathbf{1}}\right|=t_{21},\left|\mathbf{D}_{\mathbf{2 2}} \mathbf{L}_{\mathbf{2}}\right|=d_{22},\left|\mathbf{D}_{\mathbf{3 3}} \mathbf{L}_{\mathbf{1}}\right|=d_{21}$. We have the following equations:

$$
\left\{\begin{array}{l}
x_{1}^{2}+x_{2}^{2}-1=0 \\
\left(-h_{1} x_{1}+x_{3}\right)^{2}+\left(-h_{1} x_{2}+x_{4}\right)^{2}-t_{21}^{2}=0 \\
\left|m_{2}\left(h_{2} x_{1}+x_{3}-b_{1}\right)-l_{2}\left(h_{2} x_{2}+x_{4}\right)\right|-d_{22}=0 \\
\left|m_{1}\left(-h_{3} x_{2}+x_{3}-b_{2}\right)-l_{1}\left(h_{3} x_{1}+x_{4}-b_{3}\right)\right|-d_{21}=0
\end{array}\right.
$$

We may simplify the equation system as follows.

$$
\left\{\begin{array}{l}
x_{1}^{2}+x_{2}^{2}-1=0 \\
x_{3}^{2}+x_{4}^{2}-2 h_{1} x_{1} x_{3}-2 h_{1} x_{2} x_{4}+s_{1}=0 \\
s_{4} x_{1}+m_{2} x_{3}-s_{5} x_{2}-l_{2} x_{4}+s_{2}=0 \\
s_{6} x_{2}+m_{1} x_{3}-s_{7} x_{1}-l_{1} x_{4}+s_{3}=0
\end{array}\right.
$$

where $s_{1}=h_{1}^{2}-t_{1}^{2}, s_{2}=-m_{2} b_{1} \pm d_{22}, s_{3}=-m_{1} b_{2}+l_{1} b_{3} \pm d_{21}, s_{4}=m_{2} h_{2}, s_{5}=l_{2} h_{2}$, $s_{6}=-m_{1} h_{3}, s_{7}=l_{1} h_{3}$. The triangular form is

$$
\left\{\begin{array}{l}
C S[21]=\left(z_{211} x_{1}+z_{212}\right) x_{4}+z_{213} x_{1}^{2}+z_{214} x_{1}+z_{215} \\
C S[22]=\left(z_{221} x_{1}+z_{222}\right) x_{3}+z_{223} x_{1}^{2}+z_{224} x_{1}+z_{225} \\
C S[23]=\left(z_{231} x_{1}+z_{232}\right) x_{2}+z_{233} x_{1}^{2}+z_{234} x_{1}+z_{235} \\
C S[24]=z_{241} x_{1}^{4}+z_{242} x_{1}^{3}+z_{243} x_{1}^{2}+z_{244} x_{1}+z_{245}
\end{array}\right.
$$


Case PPP-LPP If the constraints are $\left|\mathbf{D}_{\mathbf{1 1}} \mathbf{B}_{\mathbf{1}}\right|=t_{31},\left|\mathbf{D}_{\mathbf{2 2}} \mathbf{B}_{\mathbf{2}}\right|=t_{32},\left|\mathbf{D}_{\mathbf{3 3}} \mathbf{L}_{\mathbf{1}}\right|=d_{31}$, we have the following equations:

$$
\left\{\begin{array}{l}
x_{1}^{2}+x_{2}^{2}-1=0 \\
\left(-h_{1} x_{1}+x_{3}\right)^{2}+\left(-h_{1} x_{2}+x_{4}\right)^{2}-t_{31}^{2}=0 \\
\left(h_{2} x_{1}+x_{3}-b_{1}\right)^{2}+\left(h_{2} x_{2}+x_{4}\right)^{2}-t_{32}^{2}=0 \\
\left|m_{1}\left(-h_{3} x_{2}+x_{3}-b_{2}\right)-l_{1}\left(h_{3} x_{1}+x_{4}-b_{3}\right)\right|-d_{31}=0
\end{array}\right.
$$

We may simplify the equation system as follows.

$$
\left\{\begin{array}{l}
x_{1}^{2}+x_{2}^{2}-1=0 \\
s_{2} x_{3}^{2}+s_{2} x_{4}^{2}-s_{5} x_{1}-s_{6} x_{3}+s_{1}=0 \\
s_{2} x_{1} x_{3}-b_{1} x_{3}-s_{7} x_{1}+s_{2} x_{2} x_{4}+s_{3}=0 \\
s_{8} x_{2}+m_{1} x_{3}-s_{9} x_{1}-l_{1} x_{4}+s_{4}=0
\end{array}\right.
$$

where $s_{1}=h_{2} h_{1}^{2}-h_{2} t_{31}^{2}+h_{1} h_{2}^{2}+h_{1} b_{1}^{2}-h_{1} t_{31}^{2}, s_{2}=h_{2}+h_{1}, s_{3}=\frac{b_{1}^{2}+h_{2}^{2}-t_{32}^{2}-h_{1}^{2}+t_{31}^{2}}{2}, s_{4}=$ $-m_{1} b_{2}+l_{1} b_{3} \pm d_{31}, s_{5}=2 h_{1} h_{2}, s_{6}=2 h_{1} b_{1}, s_{7}=b_{1} h_{2}, s_{8}=-m_{1} h_{3}, s_{9}=l_{1} h_{3}$. The triangular form is

$$
\left\{\begin{array}{l}
C S[31]=\left(z_{311} x_{1}^{3}+z_{312} x_{1}^{2}+z_{313} x_{1}+z_{314}\right) x_{4}+z_{315} x_{1}^{4}+z_{316} x_{1}^{3}+z_{317} x_{1}^{2}+z_{318} x_{1}+z_{319} \\
C S[32]=\left(z_{321} x_{1}^{3}+z_{322} x_{1}^{2}+z_{323} x_{1}+z_{324}\right) x_{3}+z_{325} x_{1}^{4}+z_{326} x_{1}^{3}+z_{327} x_{1}^{2}+z_{328} x_{1}+z_{329} \\
C S[33]=\left(z_{331} x_{1}^{2}+z_{332} x_{1}+z_{333}\right) x_{2}+z_{334} x_{1}^{3}+z_{335} x_{1}^{2}+z_{336} x_{1}+z_{337} \\
C S[34]=z_{341} x_{1}^{6}+z_{342} x_{1}^{5}+z_{343} x_{1}^{4}+z_{344} x_{1}^{3}+z_{345} x_{1}^{2}+z_{346} x_{1}+z_{345}
\end{array}\right.
$$

Case LLL-PPP If the constraints are $\left|\mathbf{B}_{\mathbf{1}} \mathbf{L}_{\mathbf{3 3}}\right|=d_{43},\left|\mathbf{B}_{\mathbf{2}} \mathbf{L}_{\mathbf{2 2}}\right|=d_{42},\left|\mathbf{B}_{\mathbf{3}} \mathbf{L}_{\mathbf{1 1}}\right|=d_{41}$, we have the following equations:

$$
\left\{\begin{array}{l}
x_{1}^{2}+x_{2}^{2}-1=0 \\
\left|\left(l_{3} x_{2}+m_{3} x_{1}\right)\left(-h_{1} x_{1}+x_{3}\right)-\left(l_{3} x_{1}-m_{3} x_{2}\right)\left(-h_{1} x_{2}+x_{4}\right)\right|-d_{43}=0 \\
\left|\left(l_{2} x_{2}+m_{2} x_{1}\right)\left(h_{2} x_{1}+x_{3}-b_{1}\right)-\left(l_{2} x_{1}-m_{2} x_{2}\right)\left(h_{2} x_{2}+x_{4}\right)\right|-d_{42}=0 \\
\left|\left(l_{1} x_{2}+m_{1} x_{1}\right)\left(-h_{3} x_{2}+x_{3}-b_{2}\right)-\left(l_{1} x_{1}-m_{1} x_{2}\right)\left(h_{3} x_{1}+x_{4}-b_{3}\right)\right|-d_{41}=0
\end{array}\right.
$$

We may simplify the equation system as follows.

$$
\left\{\begin{array}{l}
x_{1}^{2}+x_{2}^{2}-1=0 \\
s_{6} x_{2}-s_{7} x_{1}+s_{8}=0 \\
s_{1}\left(x_{2} x_{3}-x_{1} x_{4}\right)-s_{9} x_{2}-s_{10} x_{1}+s_{2}=0 \\
s_{3}\left(x_{1} x_{3}+x_{2} x_{4}\right)-s_{5} x_{2}+s_{6} x_{1}+s_{4}=0
\end{array}\right.
$$

where $s_{1}=l_{1} m_{3}-m_{2} l_{3}, s_{2}=m_{3}\left(m_{2} h_{2} \pm d_{42}\right)+m_{2}\left(m_{3} h_{1} \pm d_{43}\right), s_{3}=l_{3} m_{1}-l_{1} m_{3}$, $s_{4}=-l_{3}\left(h_{3} l_{1} \pm d_{41}\right)+l_{1}\left(m_{3} h_{1} \pm d_{43}\right), s_{6}=s_{1} m_{3} s_{5}-s_{3} l_{3} m_{3} l_{2} b_{1}, s_{7}=s_{1} m_{3} s_{6}+s_{3} l_{3} m_{3} m_{2} b_{1}$, $s_{8}=s_{3} l_{3} s_{2}-s_{1} m_{3} m 4+s_{3} s_{1}\left(-m_{3} h_{1} \pm d_{3}\right), s_{9}=m_{3} l_{2} b_{1}, s_{10}=m_{3} m_{2} b_{1}$. The triangular form is

$$
\left\{\begin{array}{l}
C S[41]=\left(z_{411} x_{1}+z_{412}\right) x_{4}+z_{413} x_{1}+z_{414} \\
C S[42]=z_{421} x_{3}+z_{422} x_{1}+z_{423} \\
C S[43]=z_{431} x_{2}+z_{432} x_{1}+z_{433} \\
C S[44]=z_{441} x_{1}^{2}+z_{442} x_{1}+z_{443}
\end{array}\right.
$$


Case LLP-PPP If the constraints are $\left|\mathbf{B}_{\mathbf{1}} \mathbf{D}_{\mathbf{1 1}}\right|=t_{51},\left|\mathbf{B}_{\mathbf{2}} \mathbf{L}_{\mathbf{2} 2}\right|=d_{52},\left|\mathbf{B}_{\mathbf{3}} \mathbf{L}_{\mathbf{1 1}}\right|=d_{51}$, we have the following equations:

$$
\left\{\begin{array}{l}
x_{1}^{2}+x_{2}^{2}-1=0 \\
\left(-h_{1} x_{1}+x_{3}\right)^{2}+\left(-h_{1} x_{2}+x_{4}\right)^{2}-t_{51}^{2}=0 \\
\left|\left(l_{2} x_{2}+m_{2} x_{1}\right)\left(h_{2} x_{1}+x_{3}-b_{1}\right)-\left(l_{2} x_{1}-m_{2} x_{2}\right)\left(h_{2} x_{2}+x_{4}\right)\right|-d_{52}=0 \\
\left|\left(l_{1} x_{2}+m_{1} x_{1}\right)\left(-h_{3} x_{2}+x_{3}-b_{2}\right)-\left(l_{1} x_{1}-m_{1} x_{2}\right)\left(h_{3} x_{1}+x_{4}-b_{3}\right)\right|-d_{51}=0
\end{array}\right.
$$

We may simplify the equation system as follows.

$$
\left\{\begin{array}{l}
x_{1}^{2}+x_{2}^{2}-1=0 \\
s_{2} x_{3}^{2}+s_{2} x_{4}^{2}+s_{11} x_{2}+s_{12} x_{1}+s_{10}=0 \\
s_{6}\left(x_{2} x_{3}-x_{1} x_{4}\right)-s_{7} x_{2}-s_{8} x_{1}+s_{9}=0 \\
s_{2}\left(x_{1} x_{3}+x_{2} x_{4}\right)+s_{3} x_{2}+s_{4} x_{1}+s_{5}=0
\end{array}\right.
$$

where $s_{1}=h_{1}^{2}-t_{51}^{2}, s_{2}=l_{2} m_{1}-m_{2} l_{1}, s_{3}=l_{2}\left(l_{1} b_{1}-l_{1} b_{2}-m_{1} b_{3}\right), s_{4}=l_{1}\left(b_{1} m_{2}+l_{2} b_{3}\right)-m_{1} l_{2} b_{2}$, $s_{5}=-l_{2} l_{1} h_{3}-l_{1} h_{2} m_{2} \pm l_{2} d_{51} \pm l_{1} d_{52}, s_{6}=-s_{2}, s_{7}=m_{2}\left(l_{1} b_{2}+m_{1} b_{3}\right)-m_{1} l_{1} b_{1}, s_{8}=$ $\left.m_{2}\left(l_{1} b_{3}+m_{1} b_{2}\right)-m_{1} m_{2} b_{1}\right), s_{9}=-m_{2} l_{1} h_{3}-m_{1} m_{2} h_{2} \pm m_{2} d_{1} \pm m_{1} d_{52}, s_{10}=s_{2} s_{1}+2 h_{1} s_{5}$, $s_{11}=2 h_{1} s_{3}, s_{12}=2 h_{1} s_{4}$. The triangular form is

$$
\left\{\begin{array}{l}
C S[51]=\left(z_{511} x_{1}+z_{512}\right) x_{4}+z_{513} x_{1}^{3}+z_{514} x_{1}^{2}+z_{515} x_{1}+z_{516} \\
C S[52]=\left(z_{521} x_{1}+z_{522}\right) x_{3}+z_{523} x_{1}^{3}+z_{524} x_{1}^{2}+z_{525} x_{1}+z_{526} \\
C S[53]=\left(z_{531} x_{1}+z_{532}\right) x_{2}+z_{533} x_{1}^{2}+z_{534} x_{1}+z_{535} \\
C S[54]=z_{541} x_{1}^{4}+z_{542} x_{1}^{3}+z_{543} x_{1}^{2}+z_{545}
\end{array}\right.
$$

Case LPP-PPP If the constraints are $\left|\mathbf{B}_{\mathbf{1}} \mathbf{D}_{\mathbf{1 1}}\right|=t_{61},\left|\mathbf{B}_{\mathbf{2}} \mathbf{D}_{\mathbf{2 2}}\right|=t_{62},\left|\mathbf{B}_{\mathbf{3}} \mathbf{L}_{\mathbf{1 1}}\right|=d_{61}$, we have the following equations:

$$
\left\{\begin{array}{l}
x_{1}^{2}+x_{2}^{2}-1=0 \\
\left(-h_{1} x_{1}+x_{3}\right)^{2}+\left(-h_{1} x_{2}+x_{4}\right)^{2}-t_{61}^{2}=0 \\
\left(h_{2} x_{1}+x_{3}-b_{1}\right)^{2}+\left(h_{2} x_{2}+x_{4}\right)^{2}-t_{62}^{2}=0 \\
\left|\left(l_{1} x_{2}+m_{1} x_{1}\right)\left(-h_{3} x_{2}+x_{3}-b_{2}\right)-\left(l_{1} x_{1}-m_{1} x_{2}\right)\left(h_{3} x_{1}+x_{4}-b_{3}\right)\right|-d_{61}=0
\end{array}\right.
$$

We may simplify the equation system as follows.

$$
\left\{\begin{array}{l}
x_{1}^{2}+x_{2}^{2}-1=0 \\
s_{2} x_{3}^{2}+s_{2} x_{4}^{2}-s_{8} x_{1}-s_{9} x_{3}+s_{1}=0 \\
s_{2}\left(x_{1} x_{3}+x_{2} x_{4}\right)-s_{10} x_{1}-b_{1} x_{3}+s_{3}=0 \\
s_{4}\left(x_{2} x_{3}-x_{1} x_{4}\right)+s_{5} x_{1}-s_{6} x_{2}+s_{11} x_{3}+s_{7}=0
\end{array}\right.
$$

where $s_{1}=h_{2} h_{1}^{2}-h_{2} t_{61}^{2}+h_{1} h_{2}^{2}+h_{1} b_{1}^{2}-h_{1} t_{61}^{2}, s_{2}=h_{2}+h_{1}, s_{3}=\frac{b_{1}^{2}+h_{2}^{2}-t_{62}^{2}-h_{1}^{2}+t_{61}^{2}}{2}, s_{4}=$ $l_{1} h_{2}+l_{1} h_{1}, s_{5}=s_{2}\left(b_{3} l_{1}-m_{1} b_{2}\right)+m_{1} b_{1} h_{2}, s_{6}=s_{2}\left(l_{1} b_{2}+m_{1} b_{3}\right), s_{7}= \pm d_{61} s_{2}-\frac{m_{1} s_{3}}{2}$, $s_{8}=2 h_{1} h_{2} b_{1}, s_{9}=2 h_{1} b_{1}, s_{10}=h_{2} b_{1}, s_{11}=m_{1} b_{1}$. The triangular form is

$$
\left\{\begin{array}{l}
C S[61]=\left(z_{611} x_{1}^{3}+z_{612} x_{1}^{2}+z_{613} x_{1}+z_{614}\right) x_{4}+z_{615} x_{1}^{4}+z_{616} x_{1}^{3}+z_{617} x_{1}^{2}+z_{618} x_{1}+z_{619} \\
C S[62]=\left(z_{621} x_{1}^{3}+z_{622} x_{1}^{2}+z_{623} x_{1}+z_{614}\right) x_{3}+z_{625} x_{1}^{4}+z_{626} x_{1}^{3}+z_{627} x_{2}+z_{628} x_{1}+z_{629} \\
C S[63]=\left(z_{631} x_{1}^{2}+z_{632} x_{1}+z_{633}\right) x_{2}+z_{634} x_{1}^{3}+z_{635} x_{1}^{2}+z_{636} x_{1}+z_{637} \\
C S[64]=z_{641} x_{1}^{6}+z_{642} x_{1}^{5}+z_{643} x_{1}^{4}+z_{644} x_{1}^{3}+z_{645} x_{1}^{2}+z_{646} x_{1}+z_{647}
\end{array}\right.
$$


Case LPP-PLL If the constraints are $\left|\mathbf{B}_{\mathbf{1}} \mathbf{L}_{\mathbf{3 3}}\right|=d_{73},\left|\mathbf{D}_{\mathbf{2 2}} \mathbf{L}_{\mathbf{2}}\right|=d_{72},\left|\mathbf{D}_{\mathbf{3 3}} \mathbf{L}_{\mathbf{1}}\right|=d_{71}$, we have

$$
\left\{\begin{array}{l}
x_{1}^{2}+x_{2}^{2}-1=0 \\
\left|\left(l_{3} x_{2}+m_{3} x_{1}\right)\left(-h_{1} x_{1}+x_{3}\right)-\left(l_{3} x_{1}-m_{3} x_{2}\right)\left(-h_{1} x_{2}+x_{4}\right)\right|-d_{73}=0 \\
\left|m_{2}\left(h_{2} x_{1}+x_{3}-b_{1}\right)-l_{2}\left(h_{2} x_{2}+x_{4}\right)\right|-d_{72}=0 \\
\left|m_{1}\left(-h_{3} x_{2}+x_{3}-b_{2}\right)-l_{1}\left(h_{3} x_{1}+x_{4}-b_{3}\right)\right|-d_{71}=0
\end{array}\right.
$$

We may simplify the equation system as follows.

$$
\left\{\begin{array}{l}
x_{1}^{2}+x_{2}^{2}-1=0 \\
m_{3}\left(x_{1} x_{3}+x_{2} x_{4}\right)+l_{3}\left(x_{2} x_{3}-x_{1} x_{4}\right)+s_{1}=0 \\
s_{4} x_{1}+m_{2} x_{3}-s_{5} x_{2}-l_{2} x_{4}+s_{2}=0 \\
s_{6} x_{2}+m_{1} x_{3}-s_{7} x_{1}-l_{1} x_{4}+s_{3}=0
\end{array}\right.
$$

where $s_{1}=-m_{3} h_{1} \pm d_{73}, s_{2}=-m_{2} b_{1} \pm d_{72}, s_{3}=-m_{1} b_{2}+l_{1} b_{3} \pm d_{71}, s_{4}=m_{2} h_{2}, s_{5}=l_{2} h_{2}$, $s_{6}=-m_{1} h_{3}, s_{7}=l_{1} h_{3}$. The triangular form is

$$
\left\{\begin{array}{l}
C S[71]=\left(z_{711} x_{1}+z_{712}\right) x_{4}+z_{713} x_{1}^{2}+z_{714} x_{1}+z_{715} \\
C S[72]=\left(z_{721} x_{1}+z_{722}\right) x_{3}+z_{723} x_{1}^{2}+z_{724} x_{1}+z_{725} \\
C S[73]=\left(z_{731} x_{1}+z_{732}\right) x_{2}+z_{733} x_{1}^{2}+z_{734} x_{1}+z_{735} \\
C S[74]=z_{741} x_{1}^{4}+z_{742} x_{1}^{3}+z_{743} x_{1}^{2}+z_{744} x_{1}+z_{745}
\end{array}\right.
$$

Case LLP-PPL If the constraints are $\left|\mathbf{B}_{\mathbf{1}} \mathbf{L}_{\mathbf{3 3}}\right|=d_{83},\left|\mathbf{B}_{\mathbf{2}} \mathbf{L}_{\mathbf{2} 2}\right|=d_{82},\left|\mathbf{D}_{\mathbf{3 3}} \mathbf{L}_{\mathbf{1}}\right|=d_{81}$, we have

$$
\left\{\begin{array}{l}
x_{1}^{2}+x_{2}^{2}-1=0 \\
\left|\left(l_{3} x_{2}+m_{3} x_{1}\right)\left(-h_{1} x_{1}+x_{3}\right)-\left(l_{3} x_{1}-m_{3} x_{2}\right)\left(-h_{1} x_{2}+x_{4}\right)\right|-d_{83}=0 \\
\left|\left(l_{2} x_{2}+m_{2} x_{1}\right)\left(h_{2} x_{1}+x_{3}-b_{1}\right)-\left(l_{2} x_{1}-m_{2} x_{2}\right)\left(h_{2} x_{2}+x_{4}\right)\right|-d_{82}=0 \\
\left|m_{1}\left(-h_{3} x_{2}+x_{3}-b_{2}\right)-l_{1}\left(h_{3} x_{1}+x_{4}-b_{3}\right)\right|-d_{81}=0
\end{array}\right.
$$

We may simplify the equation system as follows.

$$
\left\{\begin{array}{l}
x_{1}^{2}+x_{2}^{2}-1=0 \\
s_{4}\left(x_{2} x_{3}-x_{1} x_{4}\right)-s_{6} x_{2}-s_{7} x_{1}+s_{1}=0 \\
s_{5}\left(x_{1} x_{3}+x_{2} x_{4}\right)-s_{8} x_{2}-s_{9} x_{1}+s_{2}=0 \\
s_{10} x_{2}+m_{1} x_{3}-s_{11} x_{1}-l_{1} x_{4}+s_{3}=0
\end{array}\right.
$$

where $s_{1}=m_{3}\left(m_{2} h_{2} \pm d_{82}\right)+m_{2}\left(m_{3} h_{1} \pm d_{83}\right), s_{2}=l_{3}\left(m_{2} h_{2} \pm d_{82}\right)+l_{2}\left(m_{3} h_{1} \pm d_{83}\right)$, $s_{3}=l_{1} b_{3}-m_{1} b_{2} \pm d_{81}, s_{4}=m_{3} l_{2}-m_{2} l_{3}, s_{5}=l_{3} m_{2}-l_{2} m_{3}, s_{6}=m_{3} l_{2} b_{1}, s_{7}=m_{3} m_{2} b_{1}$, $s_{8}=l_{3} l_{2} b_{1}, s_{9}=l_{3} m_{2} b_{1}, s_{10}=-m_{1} h_{3}, s_{11}=l_{1} h_{3}$. The triangular form

$$
\left\{\begin{array}{l}
C S[81]=\left(z_{811} x_{1}^{2}+z_{812} x_{1}+z_{813}\right) x_{4}+z_{814} x_{1}^{3}+z_{815} x_{1}^{2}+z_{816} x_{1}+z_{817} \\
C S[82]=\left(z_{821} x_{1}^{2}+z_{822} x_{1}+z_{823}\right) x_{3}+z_{824} x_{1}^{3}+z_{825} x_{1}^{2}+z_{826} x_{1}+z_{827} \\
C S[83]=\left(z_{831} x_{1}+z_{832}\right) x_{2}+z_{833} x_{1}^{2}+z_{834} x_{1}+z_{835} \\
C S[84]=z_{841} x_{1}^{4}+z_{842} x_{1}^{3}+z_{843} x_{1}^{2}+z_{844} x_{1}+z_{845}
\end{array}\right.
$$

Case LPP-PLP If the constraints are $\left|\mathbf{B}_{\mathbf{1}} \mathbf{D}_{\mathbf{1 1}}\right|=t_{91},\left|\mathbf{B}_{\mathbf{2}} \mathbf{L}_{\mathbf{2} 2}\right|=d_{92},\left|\mathbf{D}_{\mathbf{3 3}} \mathbf{L}_{\mathbf{1}}\right|=d_{91}$, we have

$$
\left\{\begin{array}{l}
x_{1}^{2}+x_{2}^{2}-1=0 \\
\left(-h_{1} x_{1}+x_{3}\right)^{2}+\left(-h_{1} x_{2}+x_{4}\right)^{2}-t_{91}^{2}=0 \\
\left|\left(l_{2} x_{2}+m_{2} x_{1}\right)\left(h_{2} x_{1}+x_{3}-b_{1}\right)-\left(l_{2} x_{1}-m_{2} x_{2}\right)\left(h_{2} x_{2}+x_{4}\right)\right|-d_{92}=0 \\
\left|m_{1}\left(-h_{3} x_{2}+x_{3}-b_{2}\right)-l_{1}\left(h_{3} x_{1}+x_{4}-b_{3}\right)\right|-d_{91}=0
\end{array}\right.
$$


We may simplify the equation system as follows.

$$
\left\{\begin{array}{l}
x_{1}^{2}+x_{2}^{2}-1=0 \\
x_{3}^{2}+x_{4}^{2}-2 h_{1}\left(x_{1} x_{3}+x_{2} x_{4}\right)+s_{1}=0 \\
l_{2}\left(x_{2} x_{3}-x_{1} x_{4}\right)+m_{2}\left(x_{1} x_{3}+x_{2} x_{4}\right)-s_{4} x_{2}-s_{5} x_{1}+s_{2}=0 \\
s_{6} x_{2}+m_{1} x_{3}-s_{7} x_{1}-l_{1} x_{4}+s_{3}=0
\end{array}\right.
$$

where $s_{1}=h_{1}^{2}-t_{91}^{2}, s_{2}=m_{2} h_{2} \pm d_{92}, s_{3}=-m_{1} b_{2}+l_{1} b_{3} \pm d_{91}, s_{4}=l_{2} b_{1}, s_{5}=m_{2} b_{1}$, $s_{6}=-m_{1} h_{3}, s_{7}=l_{1} h_{3}$. The triangular form is

$$
\left\{\begin{array}{l}
C S[91]=\left(z_{911} x_{1}^{3}+z_{912} x_{1}^{2}+z_{913} x_{1}+z_{914}\right) x_{4}+z_{915} x_{1}^{4}+z_{916} x_{1}^{3}+z_{917} x_{1}^{2}+z_{918} x_{1}+z_{919} \\
C S[92]=\left(z_{921} x_{1}^{3}+z_{922} x_{1}^{2}+z_{923} x_{1}+z_{914}\right) x_{3}+z_{925} x_{1}^{4}+z_{926} x_{1}^{3}+z_{927} x_{2}+z_{928} x_{1}+z_{929} \\
C S[93]=\left(z_{931} x_{1}^{2}+z_{932} x_{1}+z_{933}\right) x_{2}+z_{934} x_{1}^{3}+z_{935} x_{1}^{2}+z_{936} x_{1}+z_{937} \\
C S[94]=z_{941} x_{1}^{6}+z_{942} x_{1}^{5}+z_{943} x_{1}^{4}+z_{944} x_{1}^{3}+z_{945} x_{1}^{2}+z_{946} x_{1}+z_{947}
\end{array}\right.
$$

The case PPP-PPP is solved in [19]. Liu et al reduced the problem to solve one equation of degree six and three linear equations.

\section{Ruler and Compass Construction for Basic Merge Patterns}

A constraint problem is called RC-constructible if it can be constructed with ruler and compass. When $\operatorname{CN}(\mathcal{B}, \mathcal{U})=3$, the problem can be classified into eleven different cases. Here is a summery.

1. The case dda is RC-constructible.

2. PPP-LLL and LLL-PPP are reduced to solving of one quadratic and three linear equations, and are RC-constructible.

3. PPP-LLP, LLP-PPP, LPP-PLL and LLP-PPL are reduced to solving of one quartic and three linear equations, and can not be RC-constructible respectively. We use the method proposed in [6] to decide that the roots of these quartic equations can not be constructed with ruler and compass.

4. PPP-LPP, LPP-PPP, LPP-PLP and PPP-PPP are reduced to solving of one equation of degree six and three linear equations, which are not RC-constructible respectively. The polynomials of degree six in these cases are irreducible. Then it is obvious that the roots of these equations can not be constructed with ruler and compass.

If $\operatorname{CN}(\mathcal{B}, \mathcal{U})$ is more than three, $\mathcal{U}$ is not a rigid. That is to say not all the positions and orientations of vertexes in $\mathcal{U}$ relative to each other are known. So whether the problem is $\mathrm{RC}$-constructible or not bases on both the types of constraints between $\mathcal{B}$ and $\mathcal{U}$ and those in $\mathcal{U}$.

The Case of $\mathrm{CN}(\mathcal{B}, \mathcal{U})=4$

The basic merging pattern for case $\operatorname{CN}(\mathcal{B}, \mathcal{U})=4$ is shown in Figures 8 and 9. The thin lines represent constraints between $\mathcal{B}$ and $\mathcal{U} . R_{11}$ and $R_{22}$ are rigids with three degrees of freedom. Whether the problem is RC-constructible is based on the constraint types between $\mathrm{R}$ and $\mathcal{U}, R_{11}$ and $R_{22}$. These problem can be solved with ruler and compass if there are two 


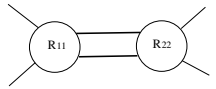

$\mathcal{U}$

Figure 8

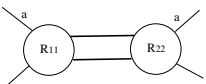

(a)

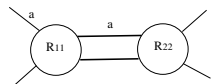

(b)

Figure 9

angle constraints, as shown in Figure 9. A line labelled 'a' represents an angle constraint, otherwise it's a distance constraint.

We make use of the fact that there are only one rotation translation between two rigids. Now the problem (a) and may be solved in two steps respectively:

1. For (a), we can get the angle constraint between $R_{11}$ and $R_{22}$ with two given angle constraints, and we use a to denote it. We position $R_{22}$ with respect to $R_{11}$ with the angle constraints a and two distances between $R_{22}$ and $R_{11}$, then we position $R_{11}$ with respect to $\mathcal{B}$. Thus the problem is reduce to two dda problems.

2. For (b), we can obtain the angle constraint between $\mathcal{B}$ and $R_{22}$ with the two given angle constraints, and we use $\mathbf{a}^{\prime}$ to denote it. First, we position $R_{22}$ with respect to $\mathcal{B}$ with the angle constraints $a^{\prime}$ and two distances between $R_{22}$ and $\mathcal{B}$, then we can position $R_{11}$ with respect to $\mathcal{B} \cup R_{22}$. Thus the problem is reduced to two dda problems.

The Case of $\operatorname{CN}(\mathcal{B}, \mathcal{U})=|V|$

If $C N(\mathcal{B}, \mathcal{U})=|V|$, according to the analysis in section $2, \mathcal{U}$ has a "ring" shaped structure (Figure 5). Each vertex $\mathrm{v}$ in $\mathcal{U}$ has exactly one constraint with primitives in $\mathcal{B}$. The constraints between objects in $\mathcal{B}$ and $\mathcal{U}$ are called external constraints.

Theorem 4.1 Let $\mathbf{L}_{\mathbf{1}}$ and $\mathbf{L}_{\mathbf{2}}$ be two adjacent lines in $\mathcal{U}$, and the external constraints for $\mathbf{L}_{1}$ and $\mathbf{L}_{\mathbf{2}}$ are an angle and a distance constraints, then $\mathcal{U}$ is $R C$-constructible.

Proof: Suppose that the external constraint for $\mathbf{L}_{\mathbf{1}}$ is an angle constraint $a_{1}$ between $\mathbf{L}_{\mathbf{1}}$ and $\mathbf{L}_{\mathbf{3}}$. Then we may obtain the angle $\angle\left(\mathbf{L}_{\mathbf{2}}, \mathbf{L}_{\mathbf{3}}\right)$ from $a_{1}$ and the angle $\angle\left(\mathbf{L}_{\mathbf{1}}, \mathbf{L}_{\mathbf{2}}\right)$. Now $\mathbf{L}_{\mathbf{3}}$ can be determined by this angle constraint and the two origin distance constraints. The rest of the primitives in $\mathcal{U}$ can be constructed explicitly, since the ring has been "broken".

Theorem 4.2 Let $\mathbf{L}_{\mathbf{1}}$ and $\mathbf{L}_{\mathbf{2}}$ be two adjacent lines in $\mathcal{U}$ and the external constraints for $\mathbf{L}_{\mathbf{1}}$ and $\mathbf{L}_{\mathbf{2}}$ be angles $a_{1}$ and $a_{2}$, then $a_{1}$ or $a_{2}$ is a redundant constraint.

Proof: If $L_{1}$ and $\mathbf{L}_{\mathbf{2}}$ are adjacent, the constraint between $\mathbf{L}_{\mathbf{1}}$ and $\mathbf{L}_{\mathbf{2}}$ must be an angle constraint. If imposing angle constraint $a_{1}$ between $L_{1}$ and $\mathbf{L}_{\mathbf{3}}$ in $\mathcal{B}$ to $\mathbf{L}_{\mathbf{1}}$ and angle constraint $a_{2}$ between $\mathbf{L}_{\mathbf{2}}$ and $L_{4}$ in $\mathcal{B}$ to $\mathbf{L}_{\mathbf{2}}$, angle constraint $a_{1}$ or $a_{2}$ is redundant if $\mathbf{L}_{\mathbf{3}}$ and $L_{4}$ are incident.

Theorem 4.3 If $|V|=2 n(n \in N)$, the maximal number of angle constraints between $\mathcal{B}$ and $\mathcal{U}$ is $n$.

Proof: Supposing the number of angle constraints is more than $\mathrm{n}$, let it be $k(>n)$. Then there are $\mathrm{k}$ lines in $V$ at least. Because $\mathcal{U}$ is a cycle, there is a pair of adjacent lines in $V$ at least. If imposing two angle constraints to the adjacent two lines, there will be redundant constraint according to theorem 4.2. When the number of lines in $\mathcal{U}$ is $\mathrm{n}$, we can keep no lines adjacent in twos, thus we can impose $\mathrm{n}$ angle constraints without redundant constraint. 
If the number of lines in $V$ is more than n, we can impose one angle constraint and one distance constraint to the pair of lines, thus the problem can be RC-constructible according to Theorem 4.1 .

Theorem 4.4 If $|V|=2 n+1(n \in N)$, the maximal number of angle constraints between $\mathcal{B}$ and $\mathcal{U}$ is $n$.

Proof: If the number of angle constraints is more than $\mathrm{n}$, there is at least one adjacent pair of lines. Thus, if imposing two angle constraints to two adjacent lines, there will be a redundant constraint according to theorem 4.2. If the number of angle constraints is $n$, the number of lines should not less than $n$. We can keep $n$ lines which are not adjacent in twos and imposing $n$ angle constraints if there are $n$ lines in $V$. If the number of lines in $V$ is

more than $\mathrm{n}$, we can impose one angle constraint and one distance constraint to the pair of lines. Thus the problem is RC-constructible according to Theorem 4.1.

\section{References}

[1] B. Brüderlin, Using Geometric Rewriting Rules for Solving Geometric Problems Symbolically, Theoretical Computer Science, 116, 291-303, 1993.

[2] L. P. Chen, X. B. Wang, X. B. Chen and J. Zhou, An Optimal Method of Bipartite Graph Matching for Underconstrained Geometry Solving, Chinese J. Computers, 23(5), 523-530, 2000

[3] J. X. Dong, J. X. Ge, Y. Gao and H. L. Li, A New Method for Constraint Satisfaction in Parametric Drafting Systems, Journal of Computer Aided Design $\&$ Computer Graphics, 9(6), 513-519, 1997

[4] C. Durand and C. M. Hoffmann, A Systematic Framework for Solving Geometric Constraints Analytically, J. of Symbolic Computation, 30(5), 493-529, 2000.

[5] X. S. Gao and S. C. Chou, Solving Geometric Constraint Systems I. A Global Propagation Approach, Computer Aided Design, 30(1), 47-54, 1998.

[6] X. S. Gao and S. C. Chou, Solving Geometric Constraint Systems II. A Symbolic Approach and Decision of Rc-constructibility, Computer-Aided Design, 30(2), 115-122, 1998.

[7] X. S. Gao, C. M. Hoffmann and W. Yang, Solving Basic Gometrci Constraint Configurations with Locus Intersection, Proc. ACM SM02, 95-104, ACM Press, New York, 2002.

[8] J. Ge, S.C. Chou and X. Gao, Geometric Constraint Satisfaction Using Optimization Methods, Computer Aided Design, 31(14), 867-879, 2000.

[9] C. M. Hoffmann, A. Lomonosov and M. Sitharam, Finding Solvable Subsets of Constraint Graphs, in LNCS, NO. 1330, Springer, Berlin Heidelberg, 163-197, 1997.

[10] R. Joan-Arinyo and A. Soto, A Correct Rule-Based Geometric Constraint Solver, Computers and Graphics, 21(5), 599-609, 1997.

[11] K. Kondo, Algebraic Method for Manipulation of Dimensional Relationships in Geometric Models, Computer Aided Design, 24(3), 141-147, 1992.

[12] G. A. Kramer, Solving Geometric Constraints Systems: A Case Study in Kinematics, MIT Press, Cambridge Massachusetts, 1992.

[13] A. V. Kumar and L. Yu, Sequential Constraint Imposition for Dimension-driven Solid Models, Computer Aided Design, 33, 475-486, 2001

[14] H. Lamure and D. Michelucci, Solving Geometric Constraints By Homotopy, IEEE Trans on Visualization and Computer Graphics, 2(1):28-34, 1996. 
[15] R. S. Latham and A. E. Middleditch, Connectivity Analysis: a Tool for Processing Geometric Constraints, Computer Aided Design, 28(11), 917-928, 1994.

[16] J. Y. Lee and K. Kim, Geometric Reasoning for Knowledge-Based Design Using Graph Representation, Computer-Aided Design, 28(10), 831-841, 1998

[17] Y. T. Li, S. M. Hu and J. G Hu, Hybrid Model of Geometric Constraint Satisfaction, Journal of Computer Research and Development, 37(10), 1233-1239, 2000

[18] V. C. Lin, D. C. Gossard and R. A. Light, Variational Geometry in Computer-Aided Design, Computer Graphics, 15(3), 171-177, 1981.

[19] H. L. Liu, T. Z. Zhang and H. S. Ding, Forward Solution of the 3-RPR Planar parallel Mechanism with Wu's Method, Journal of Beijing Institute of Technology, 20(5), 565-569, 2000

[20] J. Owen, Algebraic Solution for Geometry from Dimensional Constraints, in ACM Symp., Found of Solid Modeling, ACM Press, New York, 397-407, 1991.

[21] A. Verroust, F. Schonek and D. Roller, Rule-Oriented Method for Parameterized ComputerAided Design, Computer Aided Design, 24(10), 531-540, 1992.

[22] W. T. Wu, Basic Principles of Mechanical Theorem Proving in Geometries, Science Press, Beijing, 1984; English Version, Springer-Verlag, Berlin Heidelberg, 1994. 